\title{
¿Es posible la reconstrucción de la teoría de la educación de personas adultas integrando las perspectivas humanistas, críticas y postmodernas?
}

\author{
Is It Possible to Reconstruct the Theory of Adult Education by the Integration of the \\ Humanist, Critical, and Postmodern Perspectives?
}

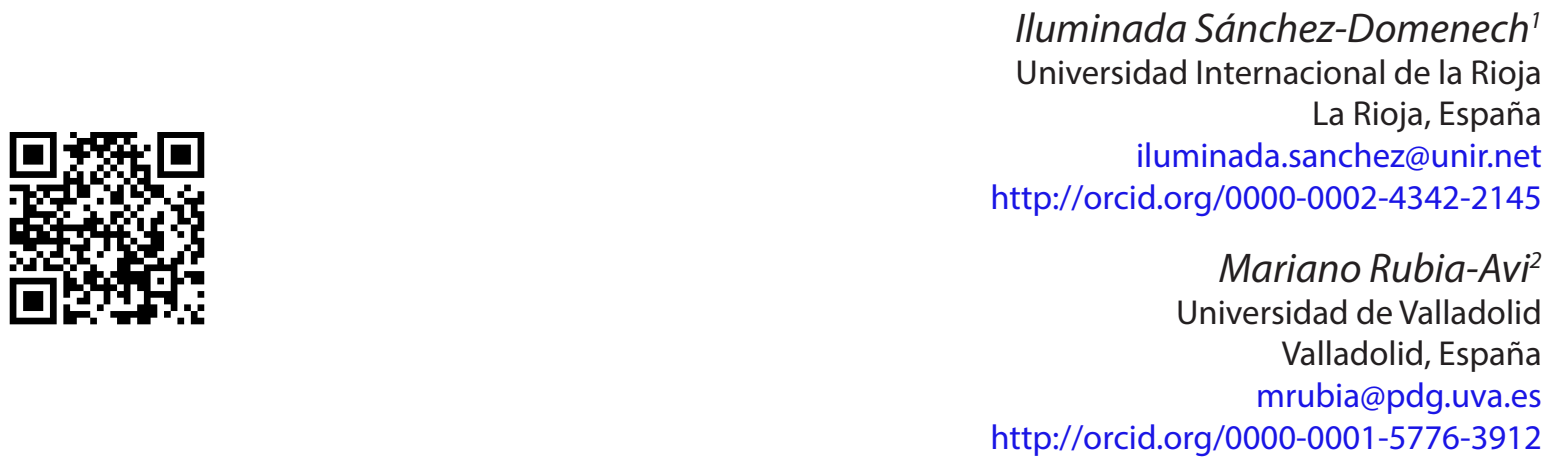

Recibido 21 de marzo de 2016 • Corregido 16 de noviembre de 2016 • Aceptado 10 de abril de 2017

Resumen: En este ensayo se trata de responder a la cuestión: ¿Es posible la integración de concepciones en principio excluyentes o contradictorias en la construcción de la teoría de la educación de adultos (EA)? Partimos de la necesidad de que la persona profesional en educación de personas adultas posea un marco teórico de referencia que le guíe en su práctica y le ayude en el desarrollo de su propia identidad como profesional. Sin embargo, las aportaciones de las distintas corrientes han tendido a ser contradictorias o excluyentes, lo que dificulta el desarrollo de la teoría. Siguiendo los objetivos de conocimiento de la teoría de la educación, analizamos algunos conceptos clave que han centrado los debates en las últimas cuatro décadas dentro del campo de estudio de la EA. Para este análisis colocamos en interacción dialéctica las corrientes educativas humanista, crítica y postmoderna/postestructuralista a través de algunos de sus autores y autoras más representativos y en torno a esos conceptos. De esta manera se muestra cómo es posible re-pensar la EA desde una perspectiva integradora, aunque con matices conceptuales e ideológicos que, lejos de ser un obstáculo, enriquecen la teoría y reflejan la diversidad de las mismas personas actoras sociales.

Palabras claves: Andragogía; educación de personas adultas; humanismo; postmodernismo; teoría de la educación; teoría crítica.

\footnotetext{
${ }^{1}$ Doctora en Pedagogía por la Universidad Cardenal Herrera-CEU. Profesora colaboradora en la Universidad Internacional de La Rioja (España). Miembro del equipo de investigación INDYCEE. Sus líneas de investigación se centran en la teoría de la educación de personas adultas.

${ }^{2}$ Doctor en Pedagogía por la Universidad de Valladolid (España), profesor titular en el Departamento de Pedagogía de la Facultad de Educación y Trabajo Social de la Universidad de Valladolid. Desde el 18 de diciembre de 2012, hasta la actualidad, es director del Departamento de Pedagogía de la Universidad de Valladolid. Sus líneas de investigación se centran en: Atención a la diversidad y educación especial.
} 
doi: http://dx.doi.org/10.15359/ree.21-2.23

URL: http://www.una.ac.cr/educare

CORREO: educare@una.cr

\begin{abstract}
This essay tries to answer the following question: is it possible the integration of apparently excluding or contradictory conceptions in the construction of the theory of adult education (AE)? We assume that there is a need for the professionals in charge of adult education to have a theoretical framework of reference that guides them in their practice, and helps them in the development of their own identity as professionals. However, the contributions of different schools of thought have tended to be contradictory or mutually excluding, hindering the development of the theory. Following the objectives of knowledge of the theory of education, we analyze some key concepts that have centered the discussions on the field of study of the AE over the past four decades. For this analysis, we create a dialectical interaction between the humanistic, critical, and postmoderm educational thoughts through some of their most representative authors and around those concepts. In this way, it is shown how it is possible to reconsider the AE from an integrative perspective, although with conceptual and ideological nuances that, far from being an obstacle, enrich the theory and reflect the diversity of the same social agents.
\end{abstract}

Keywords:Andragogy;adulteducation; critical theory; education theory; humanism; postmodernism.

Yo creo que el lenguaje hace la constante síntesis entre el horizonte del pasado y el horizonte del presente. Nos entendemos conversando, muchas veces malentendiéndonos, pero al fin y al cabo utilizando las palabras que nos hacen compartir las cosas referidas. (Gadamer, 1998, p. 61)

\title{
Introducción
}

La creciente profesionalización en educación de personas adultas en Norteamérica y Europa ha incrementado la demanda de formación de este sector planteando la necesidad de abordar la teorización y el desarrollo epistemológico de este campo de estudio. De acuerdo con Lawson (1992): "Knowing where to begin is a problem which tends to be solved historically, by selecting paradigm examples as starting point" [Saber por dónde empezar es un problema que tiende a ser resuelto históricamente seleccionando ejemplos de paradigmas como punto de partida] (p. 157). Los paradigmas o concepciones sobre el para qué y el cómo ha de ser la EA que han aportado conocimiento a la teoría de la EA son el humanismo liberal, la teoría crítica ${ }^{3}$ y el postmodernismo, cuyos correspondientes grupos seguidores han generado interesantes debates epistemológicos y conceptuales extensibles a otras etapas educativas.

Para la razón postmoderna, partir del humanismo para la construcción de una teoría de la EA no resulta aceptable. Se proclama que no es posible transgredir los límites colocados por el poder en los que se ha generado el conocimiento y en los que se halla constreñido (Foucault, 1980). Inglis (1997, p. 9) lo expresa así: "The powerful ... define not only what is good and bad, right and wrong, effective and ineffeclive, ugly and beauliful, but also what is worth knowing. Power constitules knowledge; resistance deconstructs truth" [(Los poderosos) definen no solo lo que es bueno y malo, correcto e incorrecto, eficaz e ineficaz, feo y hermoso, sino también lo que vale la pena conocer. El poder constituye el

${ }^{3}$ Corriente de la filosofía inicialmente adscrita a la Escuela de Frankfurt en la que se incluyen autores como Herbert Marcuse, Theodor Adorno, Max Horkheimer y Jürgen Habermas entre otros. 
conocimiento; la resistencia deconstruye la verdad]. La andragogía humanista de Knowles (1975, $1980,1989,1990)$ y el aprendizaje transformacional representado por Mezirow $(1981,1997,2009)$ y Kegan (2009), que se inscribe dentro de la teoría crítica representada, además, por Rikowski y McLaren (1999) en este documento, pertenecen a la categoría de esas "verdades deconstruidas" por el postmodernismo representado por Usher (1991, 2009), Usher y Bryant (1997), Inglis (1997), Biesta (2006, 2012), Edwards (2008), Zackrisson y Assarsson, (2008) y Fejes (2008).

La teoría crítica acusa al postmodernismo de la degeneración de la teoría educativa de haber traicionado al oprimido, de fragmentar la identidad de clase debilitándola en la lucha contra el capital al enfatizar las diferencias en lugar de centrarse en las similitudes, de echar por tierra el proyecto de la ilustración (Enlightenment), las meta-narrativas, la ética y los valores, así como, cualquier apelación a la "verdad". La teoría crítica hace un llamamiento a la unidad en la acción social y política en educación, en lugar de permanecer en un interminable, virtual y etéreo campo del "discurso" desde una actitud de superioridad "cool" que no lleva a ninguna parte (Rikowski y McLaren, 1999). Ante este estado de la cuestión, cabe una actitud que podríamos Ilamar "constructiva" como la adoptada por Paterson (1979), Schutz (2000), Tennant (2009), Nussbaum (2010) o Lawson (1992). Este último concuerda con la crítica postmoderna en la excesiva base psicológica de la andragogía de Knowles; pero no descarta el humanismo liberal como base para la teorización como ejemplar para lo que es la EA y para lo que debería ser.

Se hace necesario, de acuerdo con Ibáñez-Martín (2015), una "gran política", una "gran filosofía"y un "gran proyecto educativo" unidos en los mismos ideales, abarcadores de una imagen ideal del ser humano y de la sociedad, no una mera reflexión filosófica, erudita y lúcida, pero sin propuestas normativas. Sin embargo, y de acuerdo con Schutz (2000), algunos de los peligros de adoptar una teoría en su totalidad y aplicarla a cualquier tiempo y lugar de forma acrítica pueden ser mitigados teniendo acceso a un rango de diferentes e incluso (aparentemente) contradictorios marcos teóricos. Este ensayo argumenta a favor de la función de la teoría de la educación (TE) como artífice de esa unidad integradora, capaz de articular un corpus de conocimiento coherente que coloque las diferentes visiones del ser humano y el papel de la EA en la sociedad en una interacción dialéctica. Según Gargallo (2002), la TE debe contemplar algunos elementos esenciales. El autor identifica dos dimensiones en la teoría de la educación: la que describe, explica, interpreta, comprende (dimensión teórico-científica), y la que tiene por objetivo transformar la práctica (dimensión técnico-normativa). A este respecto, identifica los objetivos de conocimiento esenciales de los que nos valemos para articular este documento en sus distintos apartados. En ellos, y por razones de espacio, limitamos el análisis en torno a algunos conceptos que han centrado el debate sobre la EA desde las tres corrientes mencionadas.

\section{El contexto social y político de la educación de adultos del siglo XXI}

Uno de los elementos esenciales de la teoría de la educación es el contexto social que condiciona los fines de la educación. Los ejes conceptuales en este apartado son: 1) el aprendizaje autodirigido a lo largo de la vida; 2) individualismo frente a comunitarismo; 3) cosmopolitismo. 
doi: http://dx.doi.org/10.15359/ree.21-2.23

URL: http://www.una.ac.cr/educare

CORREO: educare@una.cr

Para Knowles (1975), uno de los objetivos conductuales de la educación que viene condicionado por la sociedad del siglo XX era el aprendizaje autodirigido a lo largo de la vida. "Autodirigido" por la necesidad psicológica del ser adulto de sentirse dueño de su vida y porque cuanto más se avanza en los niveles del sistema educativo más independencia se exige en el aprendizaje. Y "a lo largo de la vida" debido a la aceleración de la obsolescencia del ser humano, por su incapacidad para entender y hacer frente al mundo cambiante en el que vive. Esto obliga a las personas a seguir aprendiendo, a adquirir nuevos conocimientos y habilidades, actitudes, compresión y valores para seguir creciendo a lo largo de toda la vida en un mundo en constante cambio. A diferencia de Knowles, en Mezirow la autodirección no es algo instrumental. Para Mezirow (1981), la autodirección es tanto el medio como el fin de la educación de personas adultas, es tanto objetivo como método para el educador o la educadora. El aprendizaje autodirigido es la forma característica de aprender en la adultez y su filosofía distintiva.

Según Edwards (2008), el ideal de persona proyectada por el humanismo es aquella que ha asumido "as a moral obligation" [como obligación moral] (p. 31), el aprendizaje a lo largo de la vida. Desde esta perspectiva, el poder político y económico utiliza el aprendizaje a lo largo de la vida promovido por el humanismo como parte del discurso que camufla el objetivo real, es decir, el control del individuo autorresponsabilizándolo de su aprendizaje a lo largo de la vida, establecido como condición para la empleabilidad y la competitividad, elevadas ambas a la categoría de autorrealización e integración en la sociedad:

...Lifelong learning shifts responsibility from the system to the individual whereby individuals are responsible for self-emancipation and self-creation. It is the discourse of autonomous and independent individuals who are responsible for updating their skills in order to a chieve their place in society [... el aprendizaje permanente se desplaza desde la responsabilidad del sistema a la responsabilidad personal por lo cual los individuos son responsables de su auto-emancipación y auto-creación. Es el discurso de individuos autónomos e independientes que son responsables de la actualización de sus habilidades con el fin de lograr su lugar en la sociedad]. (Olssen, 2008, p. 41)

Un ejemplo que perfectamente podría justificar este juicio lo encontramos en uno de los informes de la Organización para la cooperación y desarrollo económico (OCDE, 2006): Live longer, work longer. En él se advierte que la falta de reformas dirigidas a retrasar la edad de jubilación amenaza la sostenibilidad de los presupuestos públicos, lo que forzará a una reducción drástica de la protección social. Propone algunas medidas que deben ser consideradas de forma conjunta:

1. Aumentar la inversión en el aprendizaje permanente hacia la mitad de la carrera.

2. Aumentar el atractivo de la formación y sus beneficios potenciales para los sujetos trabajadores de más edad mediante la adaptación de los métodos de enseñanza y los contenidos a sus necesidades. 
3. Promover una jubilación más tardía que, además del ahorro que supone en pensiones, elevará el potencial retorno de esa inversión a través de una expectativa mayor de los tiempos de amortización.

4. Eliminar la penalización a las empresas por despedir a trabajadores o trabajadoras de edad avanzada, pues provoca que las empresas lleguen a acuerdos para la jubilación anticipada para sustituirles por personal de menor edad. Las normas de protección del empleo son contraproducentes, la mejor forma de proteger a las personas trabajadoras de más edad es mejorar sus posibilidades de empleo y ampliar sus oportunidades de trabajo mediante la formación permanente.

Como vemos, el objetivo central es promover el aprendizaje a lo largo de la vida; pero no parece que la intención sea prevenir la incapacidad de entender y participar en el mundo, o asegurar el derecho a la autorrealización o al desarrollo máximo de potencialidades, como promulgaba el humanismo. Más bien se trataría de que la "fuerza de trabajo" resulte empleable y productiva durante más tiempo, es decir, promover el "envejecimiento activo", que en la práctica significa seguir trabajando hasta amortizar la inversión en formación y que se comience a percibir la pensión lo más tarde posible. En consecuencia, el aprendizaje a lo largo de la vida deja de ser cuestión de "Learning to Be"4 para pasar a "Learning to Be Productive and Employable" (Biesta, 2006). Esto último, no obstante, no resta veracidad a la afirmación de Knowles (1975) de que el aprendizaje a lo largo de la vida se ha convertido en una cuestión de supervivencia.

Otro tema de debate se centra en la disyuntiva individualismo vs comunitarismo (o individuo vs sociedad). Según Schutz (2000), el postmodernismo está constantemente atrapado en una paradoja de desear y al mismo tiempo temer estrategias concretas que promuevan la comunidad y la libertad, entre la tensión que conlleva el logro del poder colectivo y la pérdida de la alteridad individual que conlleva con la creación de tales colectivos. Desde la perspectiva postmoderna, el ideal de ser humano autodirigido y autónomo que defiende el humanismo es una amenaza para la cohesión social y la asociación comunitaria. Sin embargo, algunas investigaciones apuntan a que la individualidad, la autonomía y la autosuficiencia son condiciones necesarias para el desarrollo de la solidaridad social y la cooperación (Realo y Allik, 2009). Según Rothstein (2002), debemos distinguir entre el individualismo egoísta y el solidario, porque este último podría ser condición necesaria para que la gente acepte, respete y apoye a personas con diferentes valores. Es decir, la conexión establecida por el postmodernismo, entre la exaltación humanista de autogobierno y autodesarrollo y el desinterés en la participación y responsabilidad social, muestra su debilidad al establecerse una nueva relación causa/efecto entre ambos atributos y disuelve su contraposición.

\footnotetext{
${ }^{4}$ En referencia al famoso informe de la UNESCO Learning to be. The world of educaiton today and tomorrow, coordinado por Faure (1972).
} 
doi: http://dx.doi.org/10.15359/ree.21-2.23

URL: http://www.una.ac.cr/educare

CORREO: educare@una.cr

Para Knowles (1989), la autodirección es condición para una actitud crítica hacia el poder político y como argumento en este aspecto cita al personal del Departamento de Educación de Brasilia, que según él, en 1974, opinaba que: "... if we succeed in producing truly self-directed learners, they will know what to do about the goverment, and it will have no basis for exiling anybody as they did with Freire" [...si tenemos éxito en la producción de alumnos verdaderamente autodirigidos, ellos sabrán qué hacer con el gobierno, y no tendrán base para desterrar a nadie, como hicieron con Freire] (p.44). Para Mezirow (1997), tampoco existe contradicción entre autonomía y responsabilidad social, al establecer como objetivo del alumnado convertirse en un pensador autónomo socialmente responsable. Sin embargo, para este, la transformación de la perspectiva es un requisito previo a la responsabilidad social. Desde el seno del propio humanismo surge el humanismo crítico que reconoce que el humanismo tradicional deposita la confianza en la posibilidad de una sociedad democrática y justa en la autonomía y la racionalidad individuales, pero falla al no considerar la naturaleza limitante de los sistemas sociales y cómo la razón humana depende de la experiencia y la existencia en la que está enmarcada (Magill y Rodriguez, 2014-2015).

En opinión de Beck (2002), aunque la ética de la autorrealización y logro individual siguen siendo los valores característicos de esos "hijos de la libertad", del "primero yo", que quieren elegir, decidir y configurarse a sí, que "aspiran a ser autores de su vida y creadores de su identidad" (p. 13), se están superando dos posiciones tradicionalmente contrarias: "el pensar en uno mismo y el vivir para otros" creándose un individualismo "cooperativo o altruista" (p. 14) que el autor llama "individualización". Son estos "hijos de la libertad", según el autor, quienes plantearán la discusión de la posibilidad de la democracia en una era global, de la democracia cosmopolita, que tras la desregulación nacional avance hacia la regulación transnacional. Por otra parte, esos "hijos de la libertad" pueden considerarse también hijos e hijas del humanismo educativo, y quizás crítico, que ahora cuestiona las estructuras de poder. Y es que "...normalizing techniques of power not only produce obedient and predictable people but also those whom we might label disobedient and impredictable" [las técnicas normalizadas de poder no solo producen gente obediente y predecible, sino también aquellos que podemos etiquetar como desobedientes e impredecibles] (Zackrisson \& Assarsson, 2008, p. 123) y que actúan de forma desafiante.

Y no hay nada más desafiante en la sociedad en la que el "economic growth has become an intrinsic value" [crecimiento económico se ha convertido en un valor intrínseco] (Biesta, 2006, p. 175), que el postmaterialismo que parece estar aumentando entre la juventud de la periferia social (Díez-Nicolás, 2011). Quizás esta sea la revolución silenciosa, a la que aludía Inglehart (1977), que el poder económico más teme. Inglehart (1997) estableció dos parámetros para evaluar el cambio de valores en las sociedades: materialismo y postmaterialismo. El primero se caracteriza por el énfasis en la seguridad económica y personal; da prioridad al crecimiento económico, al empleo y al salario, entre otros valores. En el segundo, el énfasis se deposita en la autoexpresión y el individualismo, en las relaciones y la autorrealización en el trabajo, en la calidad de vida, y una mayor implicación política entre otros valores. La tesis inicial desarrollada por Inglehart 
(1997) mantiene que, cuando las necesidades básicas no están cubiertas, el sistema de valores tiende a ser materialista y al contrario. Según Inglehart (1997), el remplazo generacional está favoreciendo un giro hacia los valores postmaterialistas. Estoes lo que parece confirmar un estudio cualitativo realizado en España por Alonso, Fernández y Nyssen (2009) para la Agencia Nacional de Evaluación de la Calidad y Acreditación (ANECA). Según los autores, los grupos universitarios españoles no son tan proclives a la ambición profesional, la meritocracia y el sacrificio personal; muestran una actitud que los autores del informe califican como "postmaterialista". Por otra parte, es posible que existan valores emergentes que no estén representados en los ítems del cuestionario de Inglehart (1997). Por ejemplo, la individualización a la que apuntaba Beck (2002), que supera tanto el individualismo como el colectivismo y que, en la práctica, se traduce en una mayor participación en movimientos sociales o en subsistemas políticos y económicos que responden mejor a los intereses individuales y locales que los mecanismos tradicionales hegemónicos de participación política y económica (Sánchez-Domenech, 2015).

El modelo de ciudadanía que necesitaba la sociedad del siglo XX crecientemente compleja e interdependiente según Knowles (1980) era:

Citizenry with broader knowledge, less parochial values, more tolerat attitudes, and greater skill in human relations .... capable of performing increasingly complicated occupational roles and capable of creatively using more leisure time. ... that is liberated from traditional prejudices and is able to establish open, empathic, and collaborative relationships with people of all sorts [Ciudadanos con un conocimiento más amplio, menos valores localistas, actitudes más tolerantes, y una mayor habilidad en las relaciones humanas. ... Que está libre de prejuicios tradicionales y es capaz de establecer relaciones abiertas, empáticas y de colaboración con personas de todo tipo]. (p. 36)

Aunque Knowles (1980) apuntaba a "valores menos localistas" como atributo necesario de la ciudadanía del siglo XX, y señala como una de las competencias necesarias la "ciudadanía global" (1990, p. 176), conviene actualizar este concepto. Para esto recurrimos de la mano de Beck (1998) a la distinción entre globalismo y globalidad. Para el sociólogo alemán, el "globalismo", urdido por el liberalismo con fines economicistas, que desaloja o debilita el quehacer político, tiene como núcleo ideológico la desaparición de la distinción entre política y economía. Y, por otro lado, globalidad se refiere al concepto de "sociedad mundial", es decir, la totalidad de las relaciones sociales que "no están integradas dentro de la política del Estado nacional ni están determinadas, ni son determinables, a través de ésta" (pp. 32-33). Es esta"sociedad mundial" la que exige "nuevos ciudadanos del mundo" (Nussbaum, 2010, p. 26) que posean "la capacidad de trascender las lealtades nacionales y de afrontar problemas internacionales" (p. 26). Olssen (2008) propone sustituir el solitario y local aprendiz a lo largo de la vida por el concepto "unfinished cosmopolitan" [cosmopolita inacabado] (p. 44). Ambos autores articulan su propuesta para la práctica educativa 
doi: http://dx.doi.org/10.15359/ree.21-2.23

URL: http://www.una.ac.cr/educare

CORREO: educare@una.cr

en el pragmatismo de Dewey, al entender el aprendizaje como una forma de vida en la que la libertad, los valores y la participación se aprenden a través de la resolución de problemas, donde el individuo contribuye al bien común como una parte de su propio desarrollo y como un proceso de compromiso con las otras personas, y donde derechos humanos y razón se distribuyen por igual. En este modelo, señala Olssen (2008): "the learning society is a global society of engagement" [la sociedad del aprendizaje es la sociedad global de compromiso] (p.44) que ya "no longer functions as an educational ideal, but as a political one" [no funciona como una ideal educativo, sino político] (p. 45). Esto es, apela a que el compromiso no puede ser solo del individuo en solitario, ni siquiera de la globalidad social, sino que debe trascender al globalismo político y económico. Sin embargo, el aspecto emocional y moral no debe descuidarse, pues ese ciudadano o ciudadana debe ser capaz también de "imaginar con compasión las dificultades del prójimo" (Nussbaum, 2010, p. 26). La autora propone el diálogo socrático como metodología para el desarrollo del pensamiento y la argumentación crítica, junto con el arte y la literatura como medio para el desarrollo de la sensibilidad, la empatía y la tolerancia necesarias para la democracia.

\section{La persona adulta como sujeto de la educación}

Lo que tienen en común la gran variedad de actividades educativas que se incluyen bajo el nombre genérico de "educación de personas adultas" son los sujetos beneficiarios de esa educación o formación, es decir, las personas adultas como sujetos de la educación. Por tanto, conviene indagar en ambos conceptos, 1) "sujeto" y 2) "adulto", además de en el concepto de 3) "madurez", y su relación con los dos anteriores, a los que dedicamos este apartado.

El concepto de "sujeto" (subject) postmoderno se refiere a la orientación de la educación hacia estudiantes como sujetos de acción y responsabilidad, y no como objetos de intervención e influencia (Biesta, 2012). Este sujeto postmoderno es aquel que debe resistirse a la imposición de una determinada coherencia interna, de un estilo de vida y de una opinión ambos conformados por el discurso del poder. Sin embargo, Tennant (2009) recuerda que son precisamente quienes tienen un fuerte sentido de su propia identidad, quienes ofrecen el mayor potencial para la resistencia, es decir, aquellos con un yo integrado y coherente a cuya búsqueda se dirige la intervención de las tecnologías humanistas/psicológicas y críticas/sociales. Para este autor, además, en algunas de las situaciones en las que el personal educativo de personas adultas trabajan la búsqueda de un yo coherente y estable es indispensable para una práctica de la EA transformadora (por ejemplo, víctimas o individuos perpetradores de acoso sexual, de violencia de género, de discriminación racial u homófoba, etc.). La transformación para el postmodernismo consiste en interpretar ese yo coherente como un texto que puede ser reinterpretado y reevaluado. No se trata, por tanto, de descubrir el auténtico o verdadero yo a través de la reflexión sobre la experiencia, sino que esta ha de ser vista como un texto que posee lecturas alternativas y, por tanto, habría un self alternativo a ser construido (Tennant, 2009). 
Para Mezirow (2009), el aprendizaje transformacional es un proceso racional y metacognitivo de reevaluar críticamente las razones que sustentan nuestros marcos de referencia y que representan factores culturales, así como de ideología, religión, política, clase, raza, género, etc.

El concepto de "adultez" se asocia normalmente al desarrollo físico que conlleva la edad, sin embargo, lleva también implícito un sentido de perfección, de cualidades morales y de carácter a las que el estatus de adultez está íntimamente ligado (Paterson, 1979). Estas cualidades morales y capacidades personales son: prudencia, compasión, discernimiento, templanza, paciencia, fortaleza, tolerancia, objetividad y capacidad de relaciones realistas y estables. La dimensión moral de la madurez, pues, se entreteje con la cognitiva y la emocional, con el yo y con los otros seres. Por otra parte, como nos recuerda Paterson (1979), gran cantidad de personas adultas son tontas, irresponsables, débiles, impulsivas, autoengañadas, egoístas, emocionalmente obtusas, carentes de visión y sensibilidad, no poseen una suficiente autoconsciencia para que su experiencia tenga una calidad instructiva, ni generosidad suficiente para establecer conexiones reales con otros individuos. "Textos" pues, en términos postmodernos, de desagradable lectura que pueden resistirse a ser "re-interpretados". Por tanto, el sujeto de acción y responsabilidad habría que plantearlo no como un factum, sino como desideratum a cultivar, lo que refrenda la función asignada por Knowles (1980) a quien educa: ayudar al alumnado a madurar. Para ese cometido, Knowles (1980) desarrolló algunas dimensiones de la maduración (autonomía, participación activa, objetivación, racionalidad y conocimiento, responsabilidad, altruismo, tolerancia a la ambigüedad, intereses amplios, etc.). Según Paterson (1979), "The project of fostering mental autonomy is the project of helping adults to be adult" [El proyecto de promover la autonomía mental, es el proyecto de ayudar a los adultos a ser adultos] (p. 81). Sin embargo, la madurez debe, en principio al menos, suponérseles a los individuos adultos y, si no lo son, tratarlos como adultos, como propone Knowles $(1975,1980,1990)$ se espera que contribuya en mayor medida al desarrollo de su madurez que el tratarlos como niños. Knowles (1980) plantea dos "cuestiones críticas" para determinar cuándo el alumnado debe ser tratado como adulto que responde desde dos perspectivas, la social y la psicológica. Desde una perspectiva social: "a person is adult to the extent that that individual is performing social roles typically assigned by our culture to those it considers to be adults - the role of worker, spouse, parent, responsable citizen, soldier, and the like" [una persona es adulta en la medida en que desempeña roles sociales típicamente asignados por nuestra cultura a aquellos que considera adultos, como el rol de trabajador, esposo/esposa, padre/madre, ciudadano responsable, soldado, etc.] (p. 24). Knowles (1980) afirma que el desarrollo social es en el ser adulto tan importante como la maduración mental y psicológica en el niño o la niña. Para McClusky (1970), la edad adulta es un momento de crecimiento, de cambio y de integración en el que se busca constantemente equilibrio entre la cantidad de energía que se necesita y la cantidad disponible. El "margen en la vida" es la ratio entre la carga y la potencia, formadas ambas por factores externos e internos. Más potencia significa un mayor margen para participar en el aprendizaje. Al mismo tiempo, el margen para 
doi: http://dx.doi.org/10.15359/ree.21-2.23

URL: http://www.una.ac.cr/educare

CORREO: educare@una.cr

otros roles, como la familia y la vida comunitaria, se reduce para quienes han asumido, en sus vidas, la carga que supone el aprendizaje permanente para mantener su cuota de mercado y su salario. No se trata solo, por tanto, de la conformación de la subjetividad únicamente, como denuncian los estudios postmodernos, sino además, de la colonización de algo tan objetivo como el tiempo de nuestro reloj que antes pertenecía a la esfera privada y que, en países con culturas aún patriarcales, ya escaseaba para las mujeres.

En segundo lugar, desde una perspectiva psicológica: "a person is adult to the extent that that individual perceives herself or himself to be essentially responsable for her or his own life" [una persona es un adulto en la medida en que el individuo se percibe a sí mismo o a sí misma como esencialmente responsable de su propia vida] (Knowles, 1980, p. 24). Según las teorías del ciclo de vida, el ser humano avanza desde la dependencia total en la niñez hacia la independencia y autonomía crecientes, lo cual supuestamente fundamenta el principio andragógico de Knowles (1980) sobre la necesidad de autonomía del sujeto adulto en el aprendizaje en coherencia con su autonomía psicológica. Pero según Manners, Durkin y Nesdale (2004), uno de los descubrimientos más interesantes de las teorías del desarrollo del ego a lo largo la vida consiste en que solo una minoría de personas adultas progresa hacia los estados avanzados, la mayoría se estabiliza en la adultez temprana o por debajo y alcanzan únicamente una rudimentaria autoconciencia y complejidad conceptual. Kegan (1994) afirma que la mayoría de la gente es incapaz de comprender plenamente y hacer frente a las demandas más complejas de la sociedad contemporánea, operando con una sofisticación cognitiva propia de épocas anteriores. Y esto es así también para las personas pertenecientes a élites educadas.

¿Pero cuál es la causa de las personas adultas no lleguen a desarrollar la autoconciencia, la complejidad conceptual y el nivel de comprensión y responsabilidad ante la sociedad contemporánea que cabría esperarse atendiendo a su edad? La respuesta a esta cuestión, según Côté (2000), podríamos hallarla en las condiciones de la sociedad mediática y de las manipulaciones de la sociedad "persona consumidora-empresa", en la que la gente es socializada para practicar y desarrollar bajos niveles de habilidad para examinar la realidad y actuar, lo que lleva a un estado de ensoñación dentro del mundo de fantasía de la cultura popular y de la recompensa positiva por permanecer en pasividad. Desde el punto de vista psicoanalítico, según el autor, se traduce en un pobre desarrollo del sentido de conciencia y de dominio del ego (ego mastery); pero en un desarrollo elevado del sentido del placer y de la gratificación inmediata. Cuando la autonomía no va acompañada de razón, de responsabilidad moral, de pensamiento crítico, de autoconciencia y de participación activa en los asuntos del mundo, se convierte en individualismo egoísta. Por esto, la fuente del problema puede no hallarse en el planteamiento teórico de las tecnologías humanistas y críticas (Tennant, 2009), sino del desarrollo de una autonomía incompleta o inmadura fomentada en lo social y del uso que de ellas haga consciente o inconscientemente el personal educador. 


\section{El educador o educadora de personas adultas}

En esta cuestión la controversia se centra en tres aspectos: 1) "profesorado" frente a "personal facilitador de aprendizaje", 2) su papel como agente de cambio, 3) la influencia de la teoría formal en su formación y en su práctica.

Según Knowles (1980), la responsabilidad de quienes ejercen la tarea de educar a adultos y adultas, comoagentes decambio entraña implicara susclientes entresactos:(1) un análisis profundo de sus más altas aspiraciones y de los cambios requeridos para lograrlos, (2) el diagnóstico de los obstáculos a superar para lograr esos cambios; (3) la planificación de una estrategia efectiva para conseguir los resultados deseados. Su parte en este proceso es la colaboración, guía, animación, consultoría, y recurso, no la de transmisión, disciplina, juzgado y autoridad. Las influencias de Knowles (1980) en este concepto de profesorado proceden de Rogers y Dewey, principalmente. Para este último, la educación en democracia es un proceso de interacción social en el que "The teacher loses the position of external boss or dictator but takes on that of leader of group activities" [el profesor pierde la posición de jefe externo o dictador, pero asume la de líder de las actividades del grupo] (Dewey, 1938, pp. 5-6). Para Rogers (1969), la integración de lo cognitivo y lo afectivo, así como, el reconocimiento y la expresión de las emociones, es crucial para el establecimiento de una relación profesorado/alumnado que promueva un clima de respeto, libertad y confianza mutua, de humanidad, que favorezca el aprendizaje y el crecimiento.

Para Mezirow (1981), no es suficiente satisfacer necesidades, sino que se debe también ayudar al alumnado adulto a transformar su forma de pensar sobre sí mismo y su mundo. El autor señala como función central en la docencia de personas adultas el aprendizaje autodirigido. Para cumplir con este cometido, la andragogía, como perspectiva profesional de la educación de personas adultas debe, según Mezirow (1981), reducir progresivamente la dependencia del alumnado con respecto a su docente. Para esto, es necesario que el alumnado asuma una creciente responsabilidad para identificar sus necesidades, pero comprendiendo los supuestos culturales y psicológicos que influyen su percepción de esas necesidades, a definir sus objetivos de aprendizaje y los recursos que usará (incluyendo al profesorado y a otros individuos del alumnado) y a planificar su propio programa incluida la evaluación, con un enfoque de autocorrección reflexiva para aprender, para tipificar y etiquetar, para tomar perspectiva y elegir. El educador o la educadora de personas adultas, según Mezirow (1997), ha de facilitar y provocar, más que ser una autoridad en la materia. Debe ser él o ella misma un modelo de persona crítica reflexiva. Debe ir progresivamente cediendo el liderazgo al grupo y convertirse en "co-learner" [co-aprendiz] (p. 11). Knowles (1990) incorpora a su teoría el aprendizaje transformacional como uno de los roles que debe asumir el personal docente de sujetos adultos.

Desde el postmodernismo se rechaza el papel asignado por Knowles $(1975,1980,1990)$ y Mezirow $(1981,1997,2009)$ al educador o educadora por relegarlo a un papel secundario, con el efecto "lernification" (Biesta, 2012). Según este, los argumentos para acometer tal efecto se 
doi: http://dx.doi.org/10.15359/ree.21-2.23

URL: http://www.una.ac.cr/educare

CORREO: educare@una.cr

basaban en el autoritarismo de la educación tradicional y en la cantidad de conocimientos que se encuentran a disposición del estudiantado en internet. Para este, el profesorado debe enseñar, no solo facilitar o guiar el aprendizaje. Y para enseñar necesita hacer juicios sobre la prioridad y balance entre los diferentes dominios a los que se dirige la educación (cualificación, socialización y subjetivación), debe poder decidir lo que es deseable en relación con las situaciones concretas y únicas que emergen del encuentro entre sí y sus estudiantes. Según el autor, la capacidad del profesorado de realizar juicios y tomar decisiones adecuadas para cada estudiante y contexto se trata de "wisdom" (sabiduría). Knowles (1980) utiliza el término "artístico" para referirse a ese profesorado capaz de elegir para cada estudiante o grupo y la situación concreta, la teoría, metodologías, técnicas y recursos didácticos más apropiados, partiendo de sus necesidades, del contexto y de la situación concreta. La "situación" viene definida, según el autor, por la materia, la motivación estudiantil para aprender esa materia, el contexto, el conocimiento previo y la dependencia/autonomía del estudiantado respecto a su personal docente.

Eesmaa (2010) señala que la gente aprende de tresformas principalmente: 1) coleccionando hechos y conocimiento sobre el mundo que le rodea (leyendo textos, escuchando charlas, aprendiendo palabras, etc.); 2) experimentando (aprender haciendo diferentes cosas); y 3) casualmente (de gente, de los medios de comunicación, etc.). Un educador o educadora, según el autor, tiene que ser bueno en crear situaciones para que sus estudiantes aprendan de las tres formas. Cuando el humanismo educativo y el aprendizaje transformacional afirman que el profesorado es solo un recurso más, reconocen que el individuo adulto aprende en múltiples lugares, de formas variadas y de personas distintas y, en consecuencia, el "facilitador o facilitadora de aprendizaje" debe reconocer y aprovechar esa realidad para ayudar al alumnado a que aprenda a utilizar todos los recursos que se encuentran a su disposición.

Inglis (1997) señala que, desde la perspectiva del aprendizaje transformacional, el poder se reduce a obstrucciones que impiden la auténtica realización del self y el aprendizaje adulto al proceso que revela y disuelve esas obstrucciones. El autor advierte que, sin un análisis del poder, sus diferentes tipos y las formas en que opera tanto en la sociedad como en los individuos, el aprendizaje transformacional puede funcionar como una sutil forma de autocontrol en lugar de resultar emancipador. Para que adquiera esa categoría, según el autor, el educador o educadora necesita la competencia y el compromiso de leer e interpretar la vida social en términos de una lucha por el poder y, debe convertirse a sí mismo o misma, en parte de la lucha de poder de la gente oprimida. Ese análisis de las estructuras sociales y políticas que constituyen sus vidas, empieza por aquellas personas que están social y políticamente más cerca de ellos o ellas, es decir, el propio profesorado como una de esas estructuras de poder (Inglis, 1997). Esto nos parece bastante coherente, o al menos nada contradictorio, con la posición anti-jerárquica del rol facilitador andragógico. De lo que carece este último modelo es de la inclusión en su tecnología de una declaración de motivos o ideológica por parte del profesorado, verbalizada ante sus estudiantes, que haga explícita las razones por las que renuncia a una relación jerárquica entre su figura y la de su alumnado. 
En cuanto a la implicación de la teoría formal en la formación del personal educativo y en su práctica, según Usher (1991), el problema en la EA no es solo la "under-theorization", sino también, la falta de conexión entre la teoría y la práctica. El autor argumenta a favor de una teoría de la educación de personas adultas que parta de la práctica, y no al contrario. La razón de esta inversión del proceso tradicional propuesta por el postmodernismo en la teorización es que el personal docente está siempre situado, histórica y culturalmente, por lo que, aunque tengan que utilizar el conocimiento teórico universal, este será siempre interpretado a la luz de la situación concreta (Usher y Bryant, 1997). En respuesta a esta propuesta, Lawson (1992) acierta al advertir que no ha de confundirse "teoría" con "conocimiento": "Theory is not knowledege, but a predictive and exploratory framework which help us to organize data, observation, claim, and so on in order tu generate knowledge" [La teoría no es conocimiento, sino un marco predictivo y exploratorio que nos ayuda a organizar los datos, la observación, la demanda, etc., con el fin de generar conocimiento] (p. 158) y, por lo tanto, no es la teoría la que está desconectada de la práctica, en todo caso, el conocimiento generado en la praxis, este sí, de naturaleza contextualizada e ideográfica. Knowles (1990) enfatiza el papel de la teoría en docentes para hacer elecciones confiables y fundamentadas, y explicar y defender por qué hace las elecciones que hace. Por otra parte, elegir la respuesta efectiva es fácil cuando existe acuerdo en los fines, según Schön (1996), pero cuando existen paradigmas contradictorios y los fines no están claros, existe una labor de "nombrar y enfocar" para que se cree la situación en la que pueda ejecutarse el conocimiento técnico. Brookfield (1995) coincide con esta función atribuida a la teoría formal de "nombrar" la práctica que actúa iluminando los elementos generales de lo que se creían experiencias idiosincrásicas; las experiencias de colegas y la teoría formal se cruzan continuamente en una interacción dialéctica de perspectivas particulares y universales.

\section{Teorías de enseñanza-aprendizaje del sujeto adulto}

En este apartado destacamos las críticas por parte de autorías postmodernas hacia la andragogía y el aprendizaje transformacional en cuanto a: 1) su supuesta descontextualización de los procesos de enseñanza-aprendizaje; 2) el excesivo peso de la psicología en la teoría de la educación desarrolladas por aquellos modelos.

Según Illeris (2009), todo aprendizaje implica la integración de dos procesos diferentes. Algunas teorías se centran en uno de esos procesos únicamente y otras en los dos: 1) el proceso de interacción externa entre aprendiz y su entorno social, cultural o material y 2) el proceso psicológico interno de elaboración y adquisición. Pero el autor no incluye en esta clasificación la dimensión micro que implica la interacción en el aula entre personas, actividad, material didáctico y situación e ignora el potencial de transformación cultural y social de esa interacción. Otra distinción respecto a las teorías de enseñanza-aprendizaje sería posible entre aquellas que describen cómo sucede el aprendizaje y las que van más allá planteando cómo debe enseñarse (tecnología) proporcionando 
doi: http://dx.doi.org/10.15359/ree.21-2.23

URL: http://www.una.ac.cr/educare

CORREO: educare@una.cr

recomendaciones derivadas de lo anterior. Para Usher y Bryant (1997), las teorías de psicología del aprendizaje reducen el conocimiento técnico a un saber instrumental medios-fines, donde este es, más bien, un saber situado y ético. Para estos autores, el concepto de aprendizaje de la psicología terapéutica, donde podemos situar a Rogers (1969), se asemeja más a cómo entiende "aprendizaje" un sujeto educador por cuatro razones: 1) se orienta a la acción y está plagada de valores; 2) considera a la persona de forma holística; 3 ) es ideográfica y contextual; 4) reconoce la conciencia humana y el carácter reflexivo del propio conocimiento.

Knowles (1990) también se refirió a la no neutralidad de las teorías de aprendizaje: "... Theories about human behavior also carry with them assumptions about human nature, the purpose of education, and desirable values" [Las teorías sobre el comportamiento humano también conllevan principios sobre la naturaleza humana, el propósito de la educación y los valores deseables] (p. 2). Por otra parte, y aunque Knowles (1980) incorpora en su tecnología de forma ecléctica las teorías de aprendizaje que existían en ese momento (ver tabla 1), defiende el humanismo como la teoría que más se parece a la educación, por dirigirse a la persona de forma holística. Sin embargo, la teoría que se elige dependerá de los objetivos que se pretenden y, a este respecto, distingue entre "formación" (training) y "educación" (education). Además, añade a las recomendaciones de esas teorías lo que llama "clima de adultez" que caracteriza a la andragogía y que, según el autor, favorece el aprendizaje adulto.

Tabla 1: Características del clima de aprendizaje según las distintas corrientes psicológicas

\begin{tabular}{|c|c|}
\hline Escuela & Características del clima \\
\hline Conductismo & $\begin{array}{l}\text { - Aumentarla probabilidad de participación en el aprendizajemedianteaprobacióny recompensas. } \\
\text { - Permitir la práctica frecuente de los nuevos comportamientos. }\end{array}$ \\
\hline Cognitivismo & $\begin{array}{l}\text { - Orden, objetivos claramente definidos, explicación de expectativas y oportunidades, apertura } \\
\text { del sistema de comprobación y de preguntas, y retroalimentación honesta y objetiva. } \\
\text { - Aprendizaje por descubrimiento. } \\
\text { - Los errores como oportunidades de aprendizaje. }\end{array}$ \\
\hline Personalidad & $\begin{array}{l}\text { - Respeto a las diferencias individuales y culturales. } \\
\text { - Control de la ansiedad. } \\
\text { - Llentar motivaciones de logro o de afiliación según preferencias personales. } \\
\text { - Clima mentalmentes son relevantes para el aprendizaje. }\end{array}$ \\
\hline Humanismo & - Seguridad, cuidado, aceptación, confianza, respeto y comprensión. \\
\hline Teorías de campo & $\begin{array}{l}\text { - Colaboración (no competitividad), promover la lealtad al grupo, relaciones de apoyo } \\
\text { interpersonal y participación activa como norma. }\end{array}$ \\
\hline Andragogía & $\begin{array}{l}\text { - Incluye todas las características anteriores: "clima de adultez". } \\
\text { - Mutualidad e informalidad en el clima. }\end{array}$ \\
\hline
\end{tabular}

Nota: Adaptado de Sánchez-Domenech (2015, p. 463) a partir de Knowles (1990). 
Las metodologías y técnicas de enseñanza, que a veces derivan de las teorías de aprendizaje, así como, el comportamiento del profesorado "enseñan"en sí mismas"students not only pick things up from what we say but also from how we say it and how we do it" [los estudiantes no solo captan las cosas de lo que decimos sino también de cómo lo decimos y de lo que hacemos] (Biesta, 2012, p. 39). Por ejemplo, para el aprendizaje de la democracia: "an educative environment... is one that exemplifies democratic values, that practices a democratic philosophy" [un entorno educativo..., es aquel que ejemplifica valores democráticos, que practica una filosofía democrática] (Knowles, 1980, p. 67). Y en coherencia con esta idea, defiende la posibilidad de elección de los recursos de aprendizaje, respeto y tolerancia, libre expresión de ideas y sentimientos, abstenerse de inducir a la competitividad y de juzgar a otras personas, promover el aprendizaje mutuo y el intercambio de experiencias como un recurso de aprendizaje, la participación democrática en el proceso tecnológico, etc. (Knowles, 1980). Se trata, así, de promover la experiencia que establece la conexión entre los dos polos de la falsa dualidad teoría/práctica y pensar/hacer, "moral theory and moral conduct" [entre teoría moral y conducta moral] (Dewey, 1891, p. 187), por lo tanto, en esta interacción se "enseñan" no solo contenidos y habilidades, sino a ser y a estar con otros seres, a conocer y a conocerse con las demás personas.

Ese "cómo" que deriva de las teorías de enseñanza-aprendizaje provenientes de la psicología, posee un atractivo especial para la educación, dado su potencial guía para la práctica. Este hecho explicaría la preponderancia en la teoría de la educación de la psicología como disciplina fundamentante que Usher (1991) denuncia, frente a otras ciencias o disciplinas como la sociología y la filosofía. Y es que la psicología del aprendizaje puede fundamentar parte de la tecnología educativa, no los fines educativos a los que debe orientarse esta. El problema, en nuestra opinión, no es, por tanto, la presencia de la psicología del aprendizaje en la teoría de la educación, sino la ausencia de otras disciplinas en lo que respecta a la teorización de la EA y, en consecuencia, en la formación de los educadores y las educadoras.

El aprendizaje experiencial goza de gran aceptación entre las teorías de la EA. El modelo de "experiential learning" más utilizado en la EA y, al mismo tiempo, más criticado por su supuesta descontextualización, es el de Kolb (1984). El autor pretendía elaborar una teoría sobre el aprendizaje que integrase, de manera holística, la experiencia, la percepción, la cognición y el comportamiento. Es decir, intentaba superar las limitaciones del conductismo, la Gestalt y el cognitivismo. Usher (2009) advierte que la pedagogía del aprendizaje experiencial posee un potencial domesticador, porque en el contexto postmoderno, el proyecto del self no es unidireccional y gobernado por la racionalidad autónoma, sino que la identidad se construye a través de "elecciones" sobre la posesión de bienes y la búsqueda de un estilo de vida gobernado por la incentivación del deseo. Como consecuencia, la "experiencia" cuyos significados están localizados dentro de la cultura consumista impulsada por el mercado, corre el peligro de convertirse en un medio por el cual ese estilo de vida es creado y "recreado". Para evitar este 
doi: http://dx.doi.org/10.15359/ree.21-2.23

URL: http://www.una.ac.cr/educare

CORREO: educare@una.cr

efecto, la metodología del aprendizaje experiencial no debe basarse exclusivamente en modelos psicologistas de descubrir, diagnosticar, categorizar o secuenciar la experiencia individual sobre la creación artificial de experiencias compartidas a través de juegos, role-play o simulaciones, según Usher (2009). Usher (2009) propone una triangulación de la experiencia a través de: 1) una indagación de significados personales 2) los significados de los otros sujetos participantes y 3) la presencia y la influencia de los diferentes contextos y discursos en esos significados.

La propuesta de Mezirow (1981), que construye su teoría crítica sobre la teoría del conocimiento de Habermas (1971), no difiere mucho en el fondo de la de Usher (2009), como podemos comprobar en la Tabla 2.

Tabla 2: Aprendizaje transformacional

\begin{tabular}{ll}
\hline $\begin{array}{c}\text { Habermas } \\
\text { Intereses cognoscitivos } \\
\text { Existencia social }\end{array}$ & \multicolumn{1}{c}{$\begin{array}{c}\text { Mezirow } \\
\text { Funciones / Método de la EA }\end{array}$} \\
\hline Técnica / trabajo & $\begin{array}{l}\text { Esfuerzo en el análisis de tareas para establecer las habilidades requeridas, } \\
\text { comportamientos, o "competencias" } \\
\text { Práctica/interacción social }\end{array}$ \\
$\begin{array}{l}\text { Ayudar al alumnado a interpretar la forma en que él, y las otras personas con las que } \\
\text { está involucrado, construyen significados, las formas en que tipifican y etiquetan a } \\
\text { otros individuos y lo que hacen y dicen cuando sus docentes interactúan el grupo. }\end{array}$ \\
Emancipación/poder & Transformación de la perspectiva \\
\hline
\end{tabular}

Nota: Elaboración propia a partir de Mezirow (1981).

La transformación de la perspectiva pone el énfasis en los problemas reales que implican relaciones de poder materializadas en ideologías institucionalizadas que se han interiorizado en la historia psicológica de cada quien. El método consiste en guiar al alumnado hacia una compresión de los motivos incrustados en esos mitos culturales que se internalizan y sus sentimientos en referencia a ellos, lo cual revelará las necesidades sentidas y deseos, así como la forma en que se ven a sí y a sus relaciones (Mezirow, 1981). Y es que el aprendizaje que cambia no solo qué conocemos sino cómo conocemos está más cercano, según Kegan (2009), a lo que etimológicamente llamamos "educación" y es solo a este último aprendizaje al que podemos llamar "transformacional".

\section{Una reflexión sobre los fines y las competencias}

Los ejes centrales de la discusión sobre los fines se concretan en lo que debe considerarse un valor intrínseco frente a lo que solo tiene un valor instrumental, debate, por otra parte, estrechamente relacionado con la cuestión de si debe primar el desarrollo del individuo (sujeto) o la transformación de lo social. 
La educación se enmarca en un telos: "that is, by a sense of purpose - which means that teachers always need to make judgements about what is desirable in relation to the different purposes that frame their practice..." [Esto es, por un sentido de propósito - que significa que los profesores siempre necesitan hacer juicios sobre lo que es deseable en relación a diferentes propósitos que enmarcan su práctica] (Biesta, 2012, p. 36). La educación, pues, es intencional en dos sentidos: el primero se refiere a la elección de ciertos fines esenciales; el segundo, a que esos objetivos proporcionan las razones por las que se seleccionan unas actividades, y no otras, que supuestamente permiten que se alcancen esos fines. Siguiendo a Paterson (1979), para que las actividades de enseñanza-aprendizaje puedan ser calificadas como "educativas" deben formar parte de una "estrategia más amplia", que debe estar dirigida, según el humanismo, a desarrollar las potencialidades que permitan la autorrealización o plenitud de la persona. Sin embargo, todas las potencialidades no pueden ser consideradas igual de benignas, lo que obliga a realizar un juicio de valor no solo sobre aquello que es deseable, sino intrínsecamente deseable. En este sentido, y según Marín (1997, citado por Bouche, 2003), las posiciones personalista, axiológica y sociocultural, adoptadas desde la filosofía en cuanto a los fines son complementarias y confluyen en dos: (1) el pleno desarrollo personal (físico, mental, moral y emocional) y (2) la activa participación en el mundo circundante (ecológico, socioeconómico y cultural).

A Knowles (1975; 1980; 1989; 1990) se le ha acusado desde la perspectiva crítica de no definir los fines sociales que persigue. Como humanista concebía la educación como un derecho humano al máximo desarrollo de las potencialidades y a la autorrealización. En su trabajo elaborado para la UNESCO en 1983 (Knowles, 1990), establece las competencias básicas para desempeñar los roles de la vida (estudiante, ser uno mismo o ser una misma, amigo o amiga, ciudadano o ciudadana, miembro de familia, trabajador o trabajadora), que contemplan tanto la dimensión individual como la social. Knowles (1989) hizo explícita la influencia de Freire (1970) y reconoce la necesidad de que la EA contribuya a la toma de conciencia histórica y cultural incluyéndola como competencia para la ciudadanía.

Del planteamiento de Knowles (1990) podemos extraer una necesaria, y a veces obviada, relación jerárquica entre fines (autorrealización), competencias conductuales (para todos los roles de la vida) y objetivos de aprendizaje (del curso). Existe, por tanto, una "estrategia más amplia" en su teoría, más abarcadora del ser humano y de la sociedad, con la que se podrá coincidir o no. En este sentido, Mezirow (1997) denuncia que, a menudo, los objetivos de la formación de personas adultas se centran en lo práctico, en objetivos a corto plazo como ser capaz de conseguir un trabajo o una promoción o enseñar a un niño o niña a leer. Para el autor es crucial que se reconozca que las necesidades de aprendizaje pueden ser definidas como objetivos a corto plazo tanto como a largo plazo. Los objetivos a corto plazo pueden ser descritos en términos de dominio de la materia de que se trate o de logro de las competencias, o de otros objetivos relacionados con el trabajo, pero para el autor, el objetivo del alumnado es convertirse en pensador autónomo socialmente responsable. 
doi: http://dx.doi.org/10.15359/ree.21-2.23

URL: http://www.una.ac.cr/educare

CORREO: educare@una.cr

Desde la posición postmoderna, Inglis (1997) cuestiona los atributos personales ensalzados por la bibliografía psicológica como autoconsciencia, autoconfianza, asertividad, eficacia y dinamismo, que aunque pueden ser necesarios, no son suficientes, para la emancipación como fin de la educación; pueden servir para funcionar mejor dentro del sistema existente, pero no para cambiarlo. Para el autor, tanto el humanismo como el aprendizaje transformacional se dirigen al empoderamiento que es conformidad y rendición; mientras que "education for liberation and emancipation is a collective educational activity which has as its goal social and political transformation" [la educación para la liberación y la emancipación es una actividad educativa colectiva que tiene como objetivo la transformación social y política] (p. 14). Sin embargo, y de acuerdo con Paterson (1979): "There is and can be no social objective which is so indisputably desirable that its pursuit can be ordained and laid down as canonical for everyone engaged in education" [No hay ni puede haber ningún objetivo social que sea tan indiscutiblemente deseable que su persecución pueda ser ordenada y establecida como canónica para todos los que participan en la educación] (p. 173). Reconozcamos que la situación actual de la educación es o tiende a ser la cautividad perpetrada por una determinada ideología económica mediante la usurpación y la manipulación del lenguaje, el control de los contenidos y la implantación de determinadas metodologías a golpe de imposición legislativa y discursiva, como denuncian los grupos críticos y postmodernos. No obstante, cambiar una ideología por otra seguiría suponiendo la instrumentalización de la educación de la que el humanismo trataba de vacunarse a través del "Enlightment" y la teoría crítica través del pensamiento crítico y la racionalidad. Mezirow (2009, p. 96) lo expresa de este modo: "A rational epistemology of adult learning holds the promise of saving adult education from becoming, like religión, prejudice and politics, the rationalization of a vested interest to give it the appearance of cause" [Una epistemología racional del aprendizaje adulto mantiene la promesa de salvar a la EA de convertirse, como la religión, el prejuicio y la política, en la racionalización de un interés particular para darle la apariencia de causa]. Porque, sigue argumentando el autor, "principles that define reasons and determine their force may change, but rationality remains the same: judgement and action in accord with reason. A critical thinker is one who is appropriately moved by reasons" [los principios que definen las razones y determinan su fuerza pueden cambiar, pero la racionalidad permanece igual: juicio y acción de acuerdo a la razón. Un pensador crítico es aquel que es apropiadamente movido por razones] (p. 96).

El autogobierno humanista se refiere a autonomía interna (Enkrateia= self-governance), racional y moral, a una conciencia propia del self y del mundo, a la libertad de pensamiento y de afiliación. Esta libertad de pensamiento y de afiliación no es posible sin la conciencia de una o uno mismo y de las estructuras, discursos y relaciones de poder que la gobiernan y la limitan que el posestructuralismo trata de desvelar, pero para desarrollar esa conciencia bien informada es necesario seguir aprendiendo a lo largo de la vida, como bien señala Lawson (1992). 
A modo de conclusión, la incursión de la teoría crítica y el postmodernismo en la teoría de la educación implican una conciencia histórico-social y una nueva reflexión crítica sobre los medios y los fines educativos. Sin embargo, para que las actividades que se llevan a cabo en las aulas puedan considerarse realmente educativas, deben dirigirse a aquello que tiene un valor indiscutiblemente intrínseco, como es el desarrollo de una mayor conciencia de sí y del mundo, la racionalidad y la moralidad autónomas, el sentido de justicia y de responsabilidad y el Enlightment. Es importante señalar que estos principios "universalizables" no están reñidos con lo local, lo diferente o lo idiosincrásico. Muy al contrario, en la dimensión práctica, y necesariamente guiados por los anteriores, se encuentran valores como la tolerancia, la participación, la solidaridad, la empatía, el afecto, el altruismo, etc., que se materializan en la relación con la alteridad inmediata. La dicotomía entre los dos polos individuo-sociedad que, según Usher (1991), impone el humanismo al privilegiar el primero, no es tal en el plano educativo, pues la dimensión social es inherente al "pleno desarrollo del ser".

En palabras de Romero y Pérez (2012): "Si el proceso educativo no consigue formar a personas que tengan actitudes favorables para llevar a cabo una vida en común, basada en una sana convivencia, y valores para la participación en los asuntos públicos, ... habría que suprimir lo de educativo" (p. 105).

La racionalidad de una sociedad es directamente proporcional a la racionalidad de los miembros que la componen o, puesto al contrario, una sociedad formada por individuos irracionales no podrá nunca ser racional (Paterson, 1979). La persona mental y moralmente autónoma hace sus propios juicios, no abraza ciegamente las elecciones o juicios hechos por otros individuos, ya sean morales o políticos, cualidades individuales esenciales para la salud de la democracia. Por tanto, los hombres y las mujeres racionales y con autonomía no son meros medios de una sociedad racional y democrática, sino una parte esencial de ella (Paterson, 1979). El análisis de las "prácticas discursivas" que Usher (2009) propone introducir en la EA es una forma de unir esos dos polos sujeto-social. Y es que el "discurso", como nos recuerda Edwards (2008), no es solo una unidad de lenguaje, sino también de acción humana, de interacción de comunicación y de cognición. Es constitutivo de conocimiento, da forma a representaciones y acciones, modela determinadas formas de conocer el mundo y de actuar en él dentro de lo posible. Por tanto, no es algo externo, que existe independientemente del ser, sino que penetra en la subjetividad, inseparable del pensar y del hacer. Según esta posición, la pretendida racionalidad neutra del humanismo y la teoría crítica estaría en realidad cautiva dentro de los márgenes del discurso del poder y, por tanto, a su servicio. 
doi: http://dx.doi.org/10.15359/ree.21-2.23

URL: http://www.una.ac.cr/educare

CORREO: educare@una.cr

\section{Conclusiones}

La pedagogía crítica y el humanismoliberal pueden ser repensados desdela postmodernidad sin que esto implique renunciar a los progresos logrados para la educación. Esto supone en la práctica lo que Gadamer (1993) llamó "fusión de horizontes", reconstruir el horizonte pasado e integrarlo dialécticamente en el presente postmoderno para poder, así, imaginar otro más lejano. No hemos ocultado la tensión entre las distintas perspectivas, no hemos intentando una "asimilación ingenua, sino desarrollarla conscientemente" (Gadamer, 1993, p. 190). Es precisamente esa tensión la que nos hizo plantearnos la pregunta: ¿Es posible la integración de concepciones distintas en principio excluyentes o contradictorias en la construcción de la teoría de la EA?, para cuya respuesta nos acogemos a las palabras de Gadamer (1993):

El que quiere comprender puede desde luego dejar en suspenso la verdad de su referencia;... Preguntar permite siempre ver las posibilidades que quedan en suspenso. Por eso no es posible comprender la cuestionabilidad desgajándose de un verdadero preguntar, como en cambio sí es posible comprender una opinión al margen del propio opinar... El que quiera pensar tiene que preguntarse. Cuando alguien dice 'aquí cabría preguntan' [sic], esto es ya una verdadera pregunta, atenuada por prudencia o cortesía. Tal es la razón por la que todo comprender es siempre algo más que un simple revivir una opinión ajena.

Cuando se pregunta se dejan abiertas posibilidades de sentido, de manera que aquello que tenga sentido pueda introducirse en la propia opinión. (p. 231)

De acuerdo con Lawson (1992), el proceso de definir un "campo de práctica", como algunos estudios prefieren llamar a la EA, es el proceso de categorizar ejemplos a ser incluidos en ese campo y para eso es necesario partir de conceptos y principios organizadores. En educación, reflexionar, explicar y nombrar esos conceptos y principios es misión de la TE que dota de sentido y dirección a la tarea educativa; un sentido, sin embargo, necesariamente dinámico y paradigmáticamente integrador de lo valioso. Pero la misión integradora de la teoría de la educación se torna más compleja en la actual coyuntura postmoderna caracterizada por la incertidumbre de los valores, la diversidad de perspectivas y la fragmentación del conocimiento. Sin embargo, la necesidad permanece: "Los maestros necesitan [ahora], como ... en el pasado, alguna justificación teórica general de lo que hacen en sus clases, ... [para] que su trabajo [tenga un objetivo] y [un] sentido" (Moore, 1980, p. 89).

Por otra parte, la vocación transformadora de la práctica atribuida a la teoría de la educación no será si no lo es a través de la persona educadora, que precisa una imagen abarcadora del ser humano y de la sociedad fundamentada en el conocimiento multidisciplinar y pluridimensional y en la reflexión crítica, que le dé identidad a ella misma, un marco teórico que le permita interpretar la realidad y actuar de forma contextualizada: "De no hacerlo así, su tarea es perfectamente 
absurda aunque en el fuero de su conciencia no se percate de ello por andar perdido en los devaneos cotidianos" (Fullat, 2000; p. 13). Pero en último término, es el educador o la educadora quien elige una pedagogía crítica, revolucionaria y de contrabando (Rikowskiy \& McLaren, 1999) o, por el contrario, una pedagogía de la adaptación y del capital humano.

Por otra parte, las personas adultas acuden a la formación para adquirir o desarrollar conocimientos y habilidades específicas, de forma voluntaria o coaccionadas explícita o tácitamente, y en muchos de esos casos la formación está orientada al ámbito laboral. En este contexto, la introducción de fines más elevados, morales, políticos, emancipatorios, etc., solo será posible a través de la experiencia, en sentido deweyano, con otros alumnos y alumnas, y con un profesorado que fomente un ambiente en el que los valores, el pensamiento crítico, la participación, la responsabilidad y la libertad de pensamiento pueden ser una experiencia real (no simulada), y en el sentido señalado por Kegan (2009), transformadora de la perspectiva en la que no cuenta únicamente el qué sino también el cómo. En cualquier caso, tenemos que asumir que "Not all people will make good political decisions, not all people will choose according to their potential, not all people will participate in lifelong learning" [No todo el mundo tomará buenas decisiones políticas, no todo el mundo elegirá de acuerdo con su potencial, no todo el mundo participará en el aprendizaje a lo largo de la vida] (Fejes, 2008; p. 97), que, por otra parte, puede ser emancipador o instrumentalizador al mismo tiempo (Alheit, 2009).

Podemos mirar el futuro y sucumbir a la "desesperación paralizante" a la que parecen abocarnos los análisis postmodernos (Schultz, 2000) o, por el contrario, más sabios gracias a ellos, mirarlo de frente para actuar en un presente consciente.

\section{Referencias}

Alheit,P.(2009). Biographicallearning - within the new lifelong learning discourse. En K.Illeris(Ed.), Contemporary theories of learning (pp. 116-128). London: Routledge. Recuperado de http:// www.pgce.soton.ac.uk/IT/Learning/Theories/ContemporaryTheoriesofLearning\%20 Learning\%20theorists\%20in\%20their\%20own\%20words\%20-\%20Knud\%20Illeris.pdf

Alonso, L. E., Fernández, C. J. y Nyssen, J. M. (2009). El debate sobre las competencias. Una investigación educativa en torno a la educación superior y el mercado de trabajo en España. Madrid: ANECA. Recuperado de http://www.aneca.es/var/media/148145/publi competencias 090303.pdf

Beck, U. (1998). ¿Qué es la globalización? Falacias del globalismo, respuestas a la globalización. Barcelona: Paidós. Recuperado https://ocw.uca.es/pluginfile.php/1496/mod resource/ content/1/beckulrichqueeslaglobalizacion.pdf

Beck, U. (2002). La sociedad del riesgo global. Madrid: Siglo XXI. 
doi: http://dx.doi.org/10.15359/ree.21-2.23

URL: http://www.una.ac.cr/educare

CORREO: educare@una.cr

Biesta, G. (2006). What's the point of lifelong learning if lifelong learning has no point? On the democratic deficit of policies for lifelong learning. European Educational Research Journal, 5(3 y 4), 169-180. doi: https://doi.org/10.2304/eerj.2006.5.3.169

Biesta, G. (2012). Giving teaching back to education: Responding to the disappearance of the Teacher. Phenomenology \& Practice, 6(2), 35-49. Recuperado de http://www.ul.ie/eps/sites/ default/files/Biesta\%202012.pdf

Bouche, J. H. (2003). Educar para un nuevo espacio humano. Perspectivas desde la antropología de la educación. Madrid: Dykinson.

Brookfield, S. D. (1995). Becoming a critically reflective teacher. San Francisco: Jossey-Bass.

Côté, J. E. (2000). Arrested adulthood: The changing nature of maturity and identity. New York: University Press.

Dewey, J. (1891). Moral theory and practice. International Journal of Ethics, 1(2), 186-203. doi: https://doi.org/10.1086/intejethi.1.2.2375407

Dewey, J. (1938). Experience and education. New York: Macmillan.

Díez-Nicolás, J. (2011). ¿Regreso a los valores materialistas? El dilema entre seguridad y libertad en los países desarrollados. RES, 15, 9-46. Recuperado de https://dialnet.unirioja.es/ servlet/articulo?codigo $=3930038$

Edwards, R. (2008). Actively seeking subjects? En A. Fejes y K. Nicoll (Eds.), Foucault and lifelong learning. Governing the subject (pp. 21-33). Oxon: Routledge. Recuperado de http://www. forschungsnetzwerk.at/downloadpub/2008 Foucault and Lifelong Learning.pdf

Eesmaa, I. (2010). The adult educator's different roles. En T. Jääger (Ed.), The art of being an adult educator. A handbook for adult educators-to-be (pp.11-18). Copenhagen: Aarhus University, Danish School of Education. Recuperado de https://www.academia.edu/784439/The art of being an adult educator

Fejes, A. (2008). Historicizing the lifelong learner. En A. Fejes y K. Nicoll (Eds.), Foucault and lifelong learning. Governing the subject (pp. 87-99). Oxon: Routledge. Recuperado de http://www. forschungsnetzwerk.at/downloadpub/2008 Foucault and Lifelong Learning.pdf

Foucault, M. (1980). Power/knowledge: Selected interviews \& other Wittings 1972-1977. New York: Pantheon Books. Recuperado de https://monoskop.org/images/5/5d/Foucault Michel Power Knowledge Selected Interviews and Other Writings 1972-1977.pdf

Fullat, O. (2000). Filosofías de la educación. Paideia. Barcelona: Ceac.

Gadamer, H-G. (1993). Verdad y método I (5a ed.). Salamanca: Sígueme.

22 Iluminada Sánchez-Domenech y Mariano Rubia-Avi

Los artículos de la Revista Electrónica Educare del Centro de Investigación y Docencia en Educación de la Universidad Nacional, Costa Rica, se comparten bajo términos de la Licencia Creative Commons: Reconocimiento, No Comercial, Sin Obra Derivada 3.0 Costa Rica. Las autorizaciones adicionales a las aquí delimitadas se pueden obtener en el correo: educare@una.cr 
Gadamer, H-G. (1998). Verdad y método Il. Salamanca: Sígueme.

Gargallo, B. (2002). La teoría de la educación. Objeto, enfoques y contenidos. Teoría de la Educación, 14, 19-46. Recuperado de http://campus.usal.es/ revistas trabajo/index. php/1130-3743/article/viewFile/2967/3004

Habermas, J. (1971). Knowledge and human interests. Boston: Beacon Press

Ibáñez-Martín, J. A. (2015). Sentido crítico, gran política y democracia mediática. Teoría de la educación, 27(1), 53-67 doi: https://doi.org/10.14201/teoredu20152715367

Illeris, K. (2009). A comprehensive understanding of human learning. En K. Illeris (Ed.), Contemporary theories of learning (pp. 7-20). London: Routledge. Recuperado de http:// www.pgce.soton.ac.uk/IT/Learning/Theories/ContemporaryTheoriesofLearning\%20 Learning\%20theorists\%20in\%20their\%20own\%20words\%20-\%20Knud\%20Illeris.pdf

Inglehart, R. (1977). The silent revolution. Changing values and political styles among western publics. New Jersey: Princeton University Press

Inglis, T. (1997). Empowerment and emancipation. Adult Education Quarterly, 48(1), 3-17. doi: https://doi.org/10.1177/074171369704800102

Kegan, R. (1994). In over our heads: The mental demands of modern life. Cambridge, MA: Harvard University Press.

Kegan, R. (2009). What "form" transforms? A constructive-developmental approach to transformative learning. En K. Illeris (Ed.), Contemporary theories of Learning (pp. 35-52). London: Routledge. Recuperado de http://www.pgce.soton.ac.uk/IT/Learning/Theories/ ContemporaryTheories ofLearning\%20Learning\%20theorists\%20in\%20their\%20 own $\% 20$ words $\% 20-\% 20$ Knud\%20llleris.pdf

Knowles, M. S. (1975). Self-directed learning: A guide for learners and teachers. New York: Association Press.

Knowles, M. S. (1980). The modern practice of adult Education: From pedagogy to andragogy. Englewoods Cliff, NJ: Cambridge Adult Education.

Knowles, M. S. (1989). The making of an adult educator. An autobiographicaljourney. San Francisco: Jossey-Bass.

Knowles, M. S. (1990). The adult learner. A neglected species (4 ${ }^{\mathrm{a}}$ ed.). Houston: Gulf Publishing Company.

Kolb, D. A. (1984). Experiential learning: Experience as the source of learning and development. New Jersey: Prentice Hall. 
doi: http://dx.doi.org/10.15359/ree.21-2.23

URL: http://www.una.ac.cr/educare

CORREO: educare@una.cr

Lawson, K. H. (Abril-junio, 1992). Usher's 'theory and metatheory in the adult education curriculum': A comment. International Journal of Lifelong Education, 11(2), 157-159. doi: https://doi.org/10.1080/0260137920110207

Magill, K.y Rodriguez, A. (2014-2015). A critical humanist curriculum. Journal for Critical Education Policy Studies, 12(3), 204-227. Recuperado de http://scholarworks.boisestate.edu/cgi/ viewcontent.cgi?article $=1028 \&$ context $=$ esl facpubs

Manners, J., Durkin, K. y Nesdale, A. (2004). Promoting advanced ego development among adults. Journal of Adult Development, 11(1), 19-27. doi: https://doi.org/10.1023/ B:JADE.0000012524.32002.8d

McClusky, H. Y. (1970) An approach to a differential psychology of the adult potential. En S. M. Grabrowski (Ed.) Adult learning \& instruction (pp. 80-95). Siracuse, NY: ERIC Clearinghouse on Adult Education.

Mezirow, J. (1997). Transformative learning: Theory to practice. New directions for adult and continuing education, 74, 5-12. Doi https://doi.org/10.1002/ace.7401

Mezirow, J. (1981). A critical theory of adult learning and education. Adult Education, 32(1), 3-24. doi: https://doi.org/10.1177/074171368103200101

Mezirow, J. (2009). An overview on transformative learning. En K. Illeris (Ed.), Contemporary theories of learning (pp. 90-105). London: Routledge, Recuperado de http://www.pgce. soton.ac.uk/IT/Learning/Theories/ContemporaryTheoriesofLearning\%20Learning\%20 theorists\%20in\%20their\%20own\%20words\%20-\%20Knud\%20llleris.pdf

Moore, T.W. (1980). Introducción a la teoría de la educación. Madrid: Alianza Editorial. Recuperado de http://cmap.upb.edu.co/rid=1N8Q6WJW6-16BYWH4-ZFR/91609004-T-W-Moore.pdf

Nussbaum, M. C. (2010). Sin fines de lucro. Por qué la democracia necesita de las humanidades. Madrid: Katz.

Organización para la cooperación y el desarrollo económico (OCDE). (2006). Live longer, work longer. Recuperado de http://www.keepeek.com/Digital-Asset-Management/oecd/ employment/live-longer-work-longer 9789264035881-en\#.V8DPqrX0Sa4\#page49

Olssen, M. (2008). Understanding the mechanisms of neoliberal control: Lifelong learning, flexibility and knowledge capitalism. En A. Fejes y K. Nicoll (Eds.), Foucault and lifelong learning. Governing the subject (pp. 34-47). Oxon: Routledge. Recuperado de http://www. forschungsnetzwerk.at/downloadpub/2008 Foucault and Lifelong Learning.pdf 
Paterson, R.W. K. (Ed.). (1979). Values, education and the adult. London: Routledge Recuperado de $\quad$ http://www.imd.inder.cu/adjuntos/article/636/Values\%20Education\%20and\%20 the\%20Adult.pdf

Realo, A. y Allik, J. (2009). On the relationship between social capital and individualismcollectivism. Social and Personality Psychology Compass, 3(6), 871-886. doi: https://doi. org/10.1111/j.1751-9004.2009.00226.x

Rikowski, G. y McLaren, P. (1999). Posmodernism in educational theroy. En D. Hill, P. McLaren, M. Cole y G. Rikowski (Eds.), Postmodernism in educational theory: Education and the politics of human resistance (pp. 3-13). London: Tufnell Press.

Rogers, C. (1969). Freedom to learn. Ohio: Merrill.

Romero, E. y Pérez, C. (2012). Aproximación al concepto de responsabilidad de Lévinas: Implicaciones educativas. Bordón, 64(4), 99-110. Recuperado de http://recyt.fecyt.es/ index.

Rothstein, B. (2002). Sweden. Social capital in the social democratic state. The Swedish Model and Civil Society, En R. D. Putnam (Ed.), Democracies in flux. Political Culture as a Condition for Democracy (pp. 207-241). Oxford: University press.

Tennant, M. (2009). Lifelong learning as a technology of the self. En Illeris, K. (Ed.), Contemporary theories of learning (pp. 147-58). London: Routledge. Recuperado de http://www.pgce. soton.ac.uk/IT/Learning/Theories/ContemporaryTheoriesofLearning\%20Learning\%20 theorists\%20in\%20their\%20own\%20words\%20-\%20Knud\%20llleris.pdf

Sánchez-Domenech, I. (2015). La andragogía de Malcolm Knowles: Teoría y tecnología de la educación de adultos (Tesis doctoral). Elche, Alicante: Universidad Cardenal Herrera CEU. Recuperado de http://dspace.ceu.es/simple-search?query=andragog\%C3\%ADa\&submit. $\underline{x=0 \text { \&submit. } y=0}$

Schön, D. (1996). From echnical rationality to reflection-in-action. En R. Edwards, A. Hanson y P. Raggatt (Eds.), Boundaries of adult learning (Adult Learners: Education and Training) (pp. 8-30.) Londres: Routledge.

Schutz, A. (2000). Teaching freedom? Postmodern perspectives. Review of Educational Research, 70(2), 215-251. doi: https://doi.org/10.3102/00346543070002215

Faure, E. (1972) Learning to be. The world of education today and tomorrow. París: UNESCO. Recuperado de http://unesdoc.unesco.org/images/0000/000018/001801e.pdf 
doi: http://dx.doi.org/10.15359/ree.21-2.23

URL: http://www.una.ac.cr/educare

CORREO: educare@una.cr

Usher, R. (Octubre-diciembre, 1991). Theory and metatheory in the adult education curriculum. International Journal of Lifelong Education, 10(4), 305-315. doi: http://dx.doi. org/10.1080/0260137920110207

Usher, R. y Bryant, I. (1997). La educación de adultos como teoría, práctica e investigación. El triángulo cautivo ( $2^{\mathrm{a}} \mathrm{ed}$.). Madrid: Morata.

Usher, R. (2009). Experience, pedagogy, and social practices. En Illeris, K. (Ed.), Contemporary theories of Learning. London: Routledge, 169-183. Recuperado de http://www.pgce. soton.ac.uk/IT/Learning/Theories/ContemporaryTheoriesofLearning\%20Learning\%20 theorists\%20in\%20their\%20own\%20words\%20-\%20Knud\%20llleris.pdf

Zackrisson, K. S. y Assarsson, L. (2008). Adult learner identitires under construction (Cap. 9). En A. Fejes y K. Nicoll (Eds.), Foucault and lifelong learning. Governing the subject (pp. 114-125). Oxon: Routledge. Recuperado de http://www.forschungsnetzwerk.at/ downloadpub/2008 Foucault and Lifelong Learning.pdf 